\title{
Development of a Novel PPAR $y$ Ligand Screening System Using Pinpoint Fluorescence-Probed Protein
}

\author{
Hiroyuki Nagai, ${ }^{1}$ Shogo Ebisu, ${ }^{2, *}$ Ryoji Abe, ${ }^{2,3}$ Tsuyoshi Goto, ${ }^{1}$ Nobuyuki Takahashi, ${ }^{1}$ \\ Takahiro HosaKa, ${ }^{3}$ and Teruo KaWADA ${ }^{1, \dagger}$ \\ ${ }^{1}$ Laboratory for Molecular Function of Food, Division of Food Science and Biotechnology, \\ Graduate School of Agriculture, Kyoto University, Uji 611-0011, Japan \\ ${ }^{2}$ Protein Express Co., Ltd., Chiba 260-0041, Japan \\ ${ }^{3}$ School of Materials Science, Japan Advanced Institute of Science and Technology, \\ Ishikawa 923-1292, Japan
}

Received November 15, 2010; Accepted November 29, 2010; Online Publication, February 7, 2011

[doi:10.1271/bbb.100810]

The activation of peroxisome-proliferator-activated receptor- $\gamma$ (PPAR $\gamma)$, which plays a central role in adipocyte differentiation, depends on ligand-dependent co-activator recruitment. In this study, we developed a novel method of PPAR $\gamma$ ligand screening by measuring the increase in fluorescent polarization accompanied by the interaction of a fluorescent co-activator and PPAR $\gamma$. Sterol receptor co-activator-1 (SRC-1), a major PPAR $\gamma$ co-activator, was probed by fluorescent TAMRA by the Amber codon fluorescence probe method. Polarization was increased by adding PPAR $\gamma$ ligands to a solution containing labeled SRC-1 (designated TAMRA-SRC-S) and PPAR $\gamma$. The disassociation constants $(\mathrm{Kd})$ of the PPAR $\gamma$ synthesized ligands, pioglitazone (221 nM), troglitazone (83.0 nM), and 15-deoxy- $\Delta 12,14$-prostaglandin $\mathbf{J}_{2}\left(15 \mathrm{~d}-\Delta \mathbf{P G J} \mathrm{J}_{2}\right)(156 \mathrm{nM})$, were determined by this method. Farnesol $(2.89 \mu \mathrm{M})$ and bixin $(21.1 \mu \mathrm{M})$, which we have reported to be PPAR $\gamma$ ligands, increased the fluorescent polarization. Their Kd values were in agreement with the $\mathrm{ED}_{50}$ values obtained in the luciferase assay. The results indicate that the method is valuable for screening natural PPAR $y$ ligands.

Key words: peroxisome-proliferator-activated receptor$\gamma(\operatorname{PPAR} \gamma)$; co-activator; high-throughput; ligand; screening system

Peroxisome-proliferator-activated receptor- $\gamma(\operatorname{PPAR} \gamma)$, a member of the nuclear hormone receptor superfamily, plays a central role in the regulation of adipocyte differentiation. ${ }^{1)}$ Thiazolidinediones (TZDs), major insulin sensitizers, promote preadipocyte differentiation through PPAR $\gamma$ activation ${ }^{1)}$ to increase the number of small adipocytes with high insulin sensitivity and to decrease the number of large adipocytes with insulin resistance. ${ }^{2)}$ Thus, TZDs improve metabolic disorders such as systemic insulin resistance. As PPAR $\gamma$ is a valuable target for the treatment of diabetes and hyperlipidemia, research and development of PPAR $\gamma$ ligands has been aggressively performed. ${ }^{3)}$ Among the many PPAR ligand screening methods, luciferase reporter assay is a major, effective method. ${ }^{4-7)}$ It is based on the ligand-dependent modulation of nuclear-receptormediated transcription. Using it, antagonists can also be detected. We and other groups have found several endogenous and natural ligands by this method. However, including other screening methods, such as the use of the Biacore system and ELISA, currently available methods of PPAR $\gamma$ ligand screening require complex procedures such as cell culture and protein fixation. Therefore, a novel high-throughput system is desired for easier PPAR $\gamma$ ligand screening.

Nuclear-receptor mediated transcriptional regulation, including PPAR $\gamma$, depends on co-activator binding to nuclear receptors induced by ligand binding. The physiological activities of nuclear receptors differ markedly among various tissues, and depend on which co-activator binds to the nuclear receptor. Recently, focusing on differences among binding co-activators, the selective PPAR modulator (SPPARM) concept has attracted attention. The aim of SPPARM is to screen for ligands that recruit selective transcriptional cofactors such as co-activators and co-repressors of PPARs, and to express only a desired action for the treatment of disease. For example, it is expected that PPAR agonists that improve insulin resistance without causing edema or weight gain will be developed in the future, but methods based on SPPARM are not yet available.

In this study, we developed a novel ligand assay using the four-base codon fluorescence-probing method, in which a fluorescence-probed amino acid is inserted into a chosen position of a protein without denaturation, ${ }^{8-11)}$ in screening PPAR $\gamma$ ligands. In the assay developed, sterol receptor co-activator-1 (SRC-1), which plays a critical role in insulin sensitivity and diabetes by modulating for PPAR $\gamma$ activity, ${ }^{12}$ ) was used as a fluorescence-probed co-activator. This novel assay is good in three-dimensional obstacles in protein interactions and maintaining high protein stability. Here, we propose a novel high-sensitivity, high-throughput system for screening PPAR $\gamma$ ligands using the assay developed.

\footnotetext{
$\dagger$ To whom correspondence should be addressed. Tel: +81-774-38-3751; Fax: +81-774-38-3752; E-mail: fat@kais.kyoto-u.ac.jp

* Present address: HIGETA SHOYU Co., Ltd., Chiba 288-8680, Japan

Abbreviations: PPAR, peroxisome proliferators-activated receptor; SRC-1, steroid receptor co-activator-1; GST, glutathione-S-transferase; RXR, retinoid-X-receptor; Tro, troglitazone; Pio, pioglitazone; $15 \mathrm{~d}-\Delta \mathrm{PGJ}_{2}, 15$-deoxy- $\Delta 12,14$-prostaglangin $\mathbf{J}_{2}$
} 


\section{Materials and Methods}

Materials. Bixin was purchased from Nacalai Tesque (Kyoto, Japan). $15 \mathrm{~d}-\Delta \mathrm{PGJ}_{2}$ and farnesol were from Wako Pure Chemicals (Osaka, Japan). All the other chemicals were purchased from Sigma (St. Louis, MO) or Nacalai Tesque, and were guaranteed to be of reagent grade or tissue culture grade.

Preparation of fluorescence-probed $S R C-1$ proteins. The cDNA of $S R C-1$ corresponding to a PPAR-binding region (623-770, a total of 148 amino acids) was cloned from a human brain cDNA library by a PCR technique in an expression vector, pROX-FL92 (Protein Express, Chiba, Japan) using NcoI and SmaI, in which an inserted fragment is fused to the Flag tag and His tag at the $\mathrm{N}$ - and the C-terminal, respectively. The sequence of CloveDirect TAMRA (Protein Express) was inserted in the Flag tag, by which TAMRA-aminophenylalanine is introduced at the ninth amino acid of the Flag tag. The final structure of the fusion protein is shown in Fig. 1A. The TAMRA-probed SRC-1 protein was expressed using pROX-FL92-SRC-1 with CloveDirect TAMRA using the RST100 E. coli HY system, and was purified using a HiTrap-HP column (GE Healthcare, Buckinghamshire, UK). Human $\operatorname{PPAR} \gamma$ was expressed as a fusion protein with GST in $E$. coli, and was purified using a GSTrap HP column (GE Bioscience Healthcare). A fluorescence image scanner (FMBIO-III, Hitachi Software) was used to visualize the purified TAMURA-SRC-S.

Fluorescence binding assay. Fluorescence anisotropy assay was performed using a single-molecule detection system, MF20 (Olympus, Tokyo). Excitation and emission wavelengths were set at 555 and $580 \mathrm{~nm}$, respectively. All slit widths were maintained at $8 \mathrm{~nm}$. The cuvette chamber was equipped with a magnetic stirrer, and it had both temperature control and dehumidifying capabilities. The basic phenomenon, which allows fluorescence polarization measurement to be used to assess nuclear receptor binding, is the increase in fluorescence polarization of fluorescence-probed SRC-1 as it acquires the capability to bind to a high-molecular-weight molecule. A theoretically maximum increase in fluorescence polarization is expected because fluorescence-probed SRC-1 (approximate molecular weight, 18,000 $\mathrm{Da}$ ) binds to PPAR $\gamma$ (molecular weight, 83,000 Da) in the present system.

The assessment of fluorescence-probed SRC-1 binding was made directly in terms of the measured polarization. For quantitative analysis of fluorescence-probed SRC-1 binding, an equation was derived on the basis of the law of addition of polarization values for the fraction of added PPAR $\gamma$ that was bound. This approach is similar to one reported previously. ${ }^{13)}$ The fraction bound $(f B)$ is given by

$$
f B=(P M-P F) / P B-P F
$$

where $P M$ is the measured polarization for a given fluorescence-probed SRC-1/PPAR mixture, $P F$ is the measured polarization of fluorescence-probed SRC-1 in the absence of PPAR, and $P B$ is the polarization of fluorescence-probed SRC-1 when completely bound to PPAR ( $P B$ was assessed by extrapolation to an infinite PPAR concentration derived from polarization data as a function of the $\operatorname{PPAR} \gamma$ concentration). The validity of this equation depends on the assumption that there is no change in the fluorescence intensity of fluorescence-probed SRC-1-1 upon binding. Within the limits of experimental error, this assumption was verified in the present study. The amount of SRC-1 bound was assessed from the product of the total amount of fluorescence-probed SRC-1 added and the fraction of PPAR $\gamma$ bound. The buffer solution for assays was $20 \mathrm{~mm}$ Tris$\mathrm{HCl}(\mathrm{pH} 7.5), 150 \mathrm{~mm} \mathrm{NaCl}, 1 \mathrm{~mm}$ EDTA, $5 \mathrm{~mm}$ dithiothreitol, and $10 \% \mathrm{v} / \mathrm{v}$ glycerol. A mixture of $250 \mathrm{~nm}$ GST-PPAR $\gamma$ and $5 \mathrm{~nm}$ fluorescence-probed SRC-1 (TAMRA-SRC-1) was added to the well. Composite or endogenous ligands $(1-100 \mu \mathrm{M})$ were added to the GSTPPAR $\gamma$ and SRC-1 mixture. After a 1-h reaction, fluorescence polarization was monitored. All ligands were present at concentrations above their respective $\mathrm{Kd}$ values for binding to $\operatorname{PPAR} \gamma$, and also at sufficient concentrations for binding to $\mathrm{SRC}-1$. In all cases, for each titration point, anisotropy was measured successively until the fluorescence polarization stabilized. The reported values are the average of five measurements after stabilization. By Scatchard analysis, the dissociation constants of the PPAR $\gamma$-ligand complex and SRC-1 were estimated.
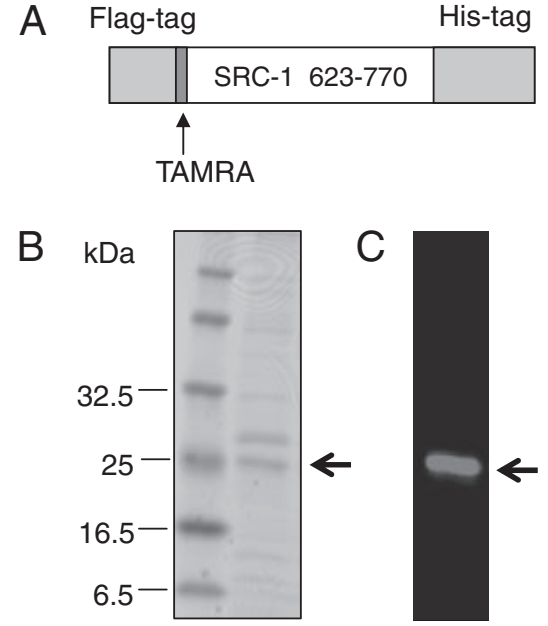

Fig. 1. Structure and Preparation of the TAMURA-SRC-S.

A, Structure of fluorescence-labeled SRC1 protein TAMRA-SRCS. B and C, Protein bands of TAMRA-SRC-S detected by CBB staining (B) and fluorescence signal $(\mathrm{C})$. The upper band in $(\mathrm{B})$ is a non-specific one. The fluorescence image of the TAMRA-SRC-S was detected by excitation $532 \mathrm{~nm}$ and at emission $605 \mathrm{~nm}$.

\section{Results}

Preparation of pin-point fluorescence-labeled SRC-S

Pin-point fluorescence-labeled SRC-S proteins were produced and purified using the RST100 E. coli HY system, as described above in "Materials and Methods." After purification using a GSTrap HP column, the fluorescent-labeled SRC-S protein, which is designated TAMRA-SRC-S, was analyzed by SDS-PAGE (Fig. 1B). The fluorescent signal of the TAMRA-SRC$\mathrm{S}$ was detected, corresponding to a CBB-stained protein band (Fig. 1C).

Kd value in binding of SRC-1 to PPAR $\mathrm{P}$ in the presence and the absence of a ligand

To examine fluorescence polarization changes accompanied by the ligand-dependent recruitment of TAMRA-SRC-S on PPAR $\gamma$, we performed binding assay to examine SRC-1 binding to PPAR $\gamma$ at various PPAR $\gamma$ concentrations ( 1 to $250 \mathrm{nM}$ ), but at a constant troglitazone concentration (Tro, $380 \mathrm{nM}$ ). In the presence of GST proteins alone as negative control, the fluorescence polarization did not increase. As shown in Fig. 2A, the fluorescence polarization increased depending on the PPAR $\gamma$ concentration in the absence and the presence of Tro. The amount of increase in the presence of Tro was stronger than that in the absence of Tro (Fig. 2B). The disassociation constants (Kd) estimated by Scatchard plot analysis were 36.3 and $73.8 \mathrm{~nm}$ in the presence, and absence, respectively, of Tro. These results indicate that the increase in fluorescence polarization was due to the specific binding of SRC-1 to $\operatorname{PPAR} \gamma$.

$K d$ values of various ligands in the binding of SRC-1 to PPAR

Next, the effects of various ligands on fluorescence polarization were examined. As PPAR ligands, we used well-known synthesized and endogenous ones: Tro, pioglitazone (Pio), and 15-deoxy- $\Delta$ 12,14-prostaglangin 
A

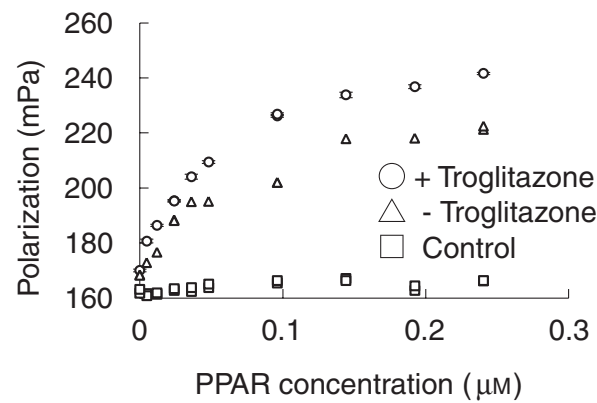

B

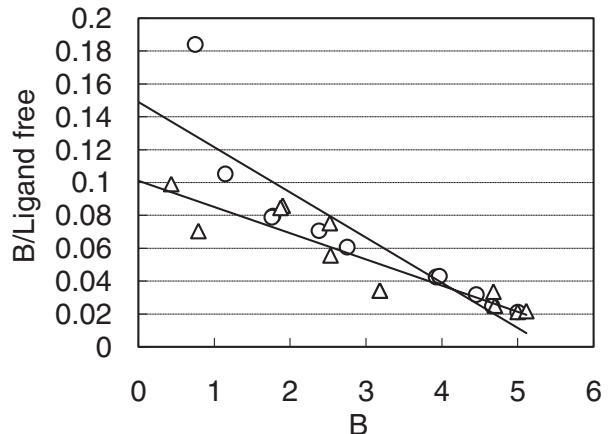

C

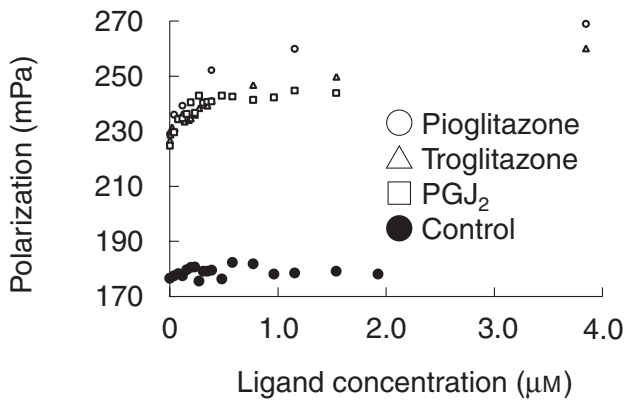

D

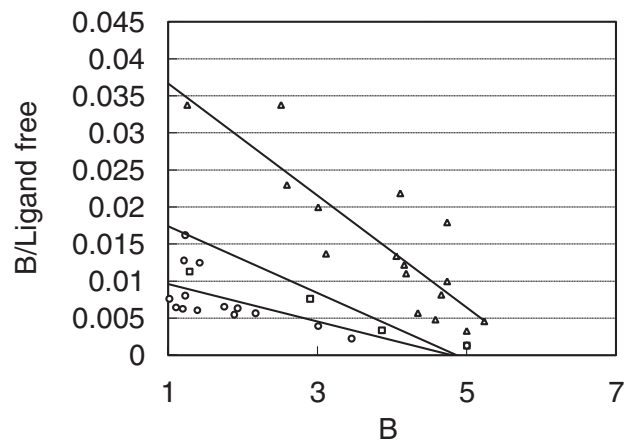

Fig. 2. Ligand-Dependent Specific Interaction between the PPAR $\gamma$-Ligand Complex and TAMRA-SRC-S.

A, Ligand-dependent changes in fluorescence polarization. TAMRA-SRC-S (5 nM) was incubated with GST-human PPAR $\gamma$ (250 nM) in the presence (open circles) or absence (open triangles) of $385 \mathrm{~nm}$ Tro. As a control experiment, TAMRA-SRC-S (5 nM) was incubated with GST protein $(250 \mathrm{~nm})$ and Tro, as indicated by open squares. After a 1-h reaction, the fluorescence polarization was measured for $10 \mathrm{~s}$. The data are representative of five independent blots. All the values are means \pm SEM for three tests. B, Scatchard plot analysis of the binding of a PPAR $\gamma$ ligand complex to the TAMRA-SRC-S in the presence (open circles) and the absence (open squares) of Tro ( $385 \mathrm{nM})$. C, Ligand-dose-dependent changes in fluorescence polarization. TAMRA-SRC-S (5 nM) was incubated with GST-human PPAR $\gamma(250 \mathrm{nM})$ and each of the following compounds (1-4,000 nM): Tro (open triangles), Pio (open circles), and $15 \mathrm{~d}-\Delta \mathrm{PGJ}_{2}$ (open squares). As a control experiment, the TAMRA-SRC-S $(5 \mathrm{nM})$ was incubated with GST protein $(250 \mathrm{nM})$ and $15 \mathrm{~d}-\Delta \mathrm{PGJ}_{2}(1-2,000 \mathrm{nM})$, as indicated by solid circles. After a $1-\mathrm{h}$ reaction, the fluorescence polarization was measured for $10 \mathrm{~s}$. The data are representative of five independent blots. All the values are means \pm SEM for three tests. D, Scatchard plot analysis of the binding of a PPAR $\gamma$-ligand complex to TAMRA-SRC-S. Plots of Tro (open triangles), Pio (open circles), and $15 \mathrm{~d}-\Delta \mathrm{PGJ}_{2}$ (open squares) are shown.

$\left(15 \mathrm{~d}-\Delta \mathrm{PGJ}_{2}\right)$. As shown in Fig. $2 \mathrm{C}$, the fluorescence polarization increased with the addition of each ligand, in a dose-dependent manner. Scatchard plot analysis revealed that a straight line could be drawn for each ligand (Fig. 2D). The $\mathrm{Kd}$ values calculated using the Scatchard plot were estimated as follows: $83.0 \mathrm{~nm}$ for Tro, $221 \mathrm{~nm}$ for Pio, and $156 \mathrm{~nm}$ for $15 \mathrm{~d}-\Delta \mathrm{PGJ}_{2}$. The number of binding sites was estimated using a hill plot (data not shown). The degree of leaning was nearly 1 for the ligand, indicating that a molecule of the ligand binds to one protein of PPAR $\gamma$. This indicates that the recruitment of TAMRA-SRC-S onto PPAR $\gamma$ is specific for ligand binding, suggesting that the fluorescence polarization change is useful in detecting the ligand$\operatorname{PPAR} \gamma$ interaction.

Evaluation of food-derived compounds by measurement of fluorescence polarization

To evaluate this novel method as a system for PPAR $\gamma$ ligand screening, natural compounds were examined by it. As a positive control, Pio was used. The fluorescence polarization was measured at various concentrations of PPAR $\gamma$ ligands (200 to $40,000 \mathrm{~nm}$ ) in the presence of PPAR $\gamma$ and of SRC-1. As shown in Fig. 3A, the addition of farnesol and bixin increased the fluorescence polarization. Scatchard plot analysis revealed that the $\mathrm{Kd}$ values were 2.89 and $21.1 \mu \mathrm{M}$ for farnesol and of bixin, respectively, although each signal was weaker than that of Pio.

\section{Discussion}

Improvements in fluorescent probe and photodetection techniques are becoming more and more important in biochemical studies. This is because fluorescent signals are easily detected and multisignals with different excitation/emission wavelengths are available. However, the sizes of recently used fluorescent molecules, such as green fluorescent protein (GFP), are large. ${ }^{14)}$ Therefore, fluorescent molecules can inhibit intermolecular bindings such as protein-protein and protein-DNA binding. Moreover, even in the case of small fluorescent molecules, it is difficult to introduce them into the desired positions. ${ }^{15,16)}$ In this context, the four-base codon fluorescence-probing method meets both requirements: low molecular weight $(700 \mathrm{Da})$ and ease of pinpoint probing. Thus, the method is valuable for detecting molecular interactions.

Ligand-dependent interactions between PPAR $\gamma$ and co-activators are indispensable for $\operatorname{PPAR} \gamma$-induced mRNA transcription. Indeed, adipocyte differentiation, which requires PPAR $\gamma$ activity, is suppressed by the ribozyme-mediated knockdown of the cAMP-response element-binding protein (CREB)-binding protein (CBP) or p300, the main co-activator for $\operatorname{PPAR} \gamma$, as previously described. ${ }^{17)}$ Moreover, PPAR $\gamma$ target genes are involved in the development of diabetes and hyperlipidemia. ${ }^{18,19)}$ Hence, it is important to develop a method of examining the interaction between PPAR $\gamma$ and its 
A

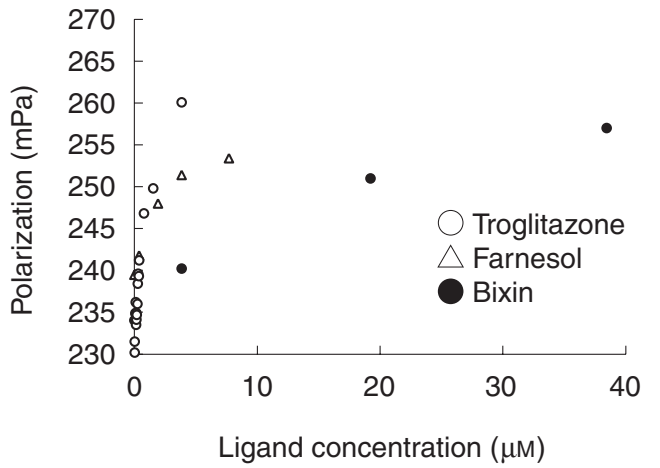

B

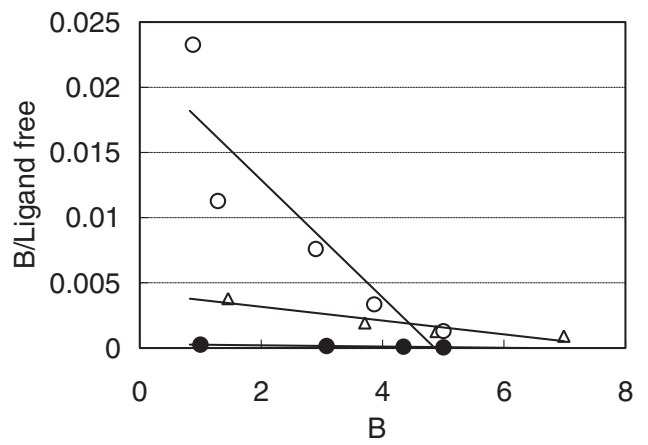

Fig. 3. Evaluation of Food-Derived Compounds as PPAR $\gamma$ Ligands by the Novel Method.

A, Dose-dependent changes in fluorescence polarization using food-derived compounds. TAMRA-SRC-S (5 nM) was incubated with GST-human PPAR $\gamma(250 \mathrm{nM})$ and each natural ligand (1$40,000 \mathrm{~nm}$ ). Tro (open circles), farnesol (open triangles), and bixin (closed circles) were used as ligands. After a 1-h reaction, the fluorescence polarization was measured for $10 \mathrm{~s}$. The data are representative of five independent blots. All the values are means \pm SEM for three tests. B, Scatchard plot analysis of the binding of a PPAR $\gamma$-ligand complex to TAMRA-SRC-S. Plots of Tro (open circles), farnesol (open squares), and bixin (open triangles) are shown.

co-activators. Co-activator pull-down assay is a valuable method for examining such an interaction in vitro, but this pull-down assay has problems, such as difficulty in quantification and low detection sensitivity. Here we report a novel quantitative method of examining the co-activator recruitment activity of PPAR $\gamma$ ligands. This method is highly sensitive and easy to use in quantifying the interaction. The $\mathrm{Kd}$ values calculated using the interaction between PPAR $\gamma$ and SRC-1 found in the present study are lower than those in previous reports. For example, it has been reported that the $\mathrm{Kd}$ values of Tro and $15 \mathrm{~d}-\Delta \mathrm{PGJ}_{2}$ are $300 \mathrm{~nm}$ and $11 \mu \mathrm{M}$, respectively. ${ }^{20)}$ The differences might be due to cellular processes such as the uptake and degradation of ligand compounds. The novel method is an in vitro assay that entails no cellular processes, whereas cell-based assays, such as reporter assay, are dependent on such processes. Therefore, the difference in $\mathrm{Kd}$ values could lead to speculations about the cellular processes specific to each ligand.

Here we found that farnesol and bixin, components of natural plants, induce interaction of $\operatorname{PPAR} \gamma$ and its co-activator, SRC-1. Previously, we found that farnesol and bixin activate PPAR $\gamma$ in luciferase assay. ${ }^{7,21)}$ The addition of farnesol or bixin induces mRNA expression of several PPAR $\gamma$ target genes in 3T3-L1 adipocytes as an agonist of $\operatorname{PPAR} \gamma$. The relative $\mathrm{Kd}$ values of Tro
$(83.0 \mathrm{nM})$, farnesol $(2.89 \mu \mathrm{M})$, and bixin $(21.1 \mu \mathrm{M})$ are similar to the ED50s obtained in luciferase assay. ${ }^{7,22)}$ Therefore, it is assumed that this method is valuable for examining the moderate effects of natural compounds on PPAR $\gamma$ activation. In addition, it enables highthroughput screening of unknown PPAR $\gamma$ ligands using micro plates. Other assay systems require operations that take a longer time, such as protein fixation and cell culture. ${ }^{3,4,19,22)}$ In the novel method, it takes approximately $2 \mathrm{~h}$ for the reaction to finish in a mixture of $\operatorname{PPAR} \gamma$, SRC-1, and a ligand candidate. Hence, it is expected that the processing time is shortened in comparison with other assay systems. These advantages of our method are indispensable for high-throughput screening of food-derived compounds serving as PPAR $\gamma$ ligands with moderate effects.

In conclusion, a novel method of examining the interaction between PPAR $\gamma$ and fluorescence-labeled SRC-1 is useful in the screening PPAR $\gamma$ ligands due to its high sensitivity and ease of operation. In addition, it may contribute to the elucidation of the various effects of co-activators with respect to the SPPARM concept, which may result in the development of novel therapies for diabetes and obesity.

\section{Acknowledgment}

We would like to thank Y. Tada for technical assistance. This work was largely supported by Grantsin-Aid for Scientific Research from the Ministry of Education, Culture, Sports, Science, and Technology of Japan (22228001 and 22380075).

\section{References}

1) Spiegelman BM and Flier JS, Cell, 87, 377-389 (1996).

2) Yamauchi T, Kamon J, Waki H, Murakami K, Motojima K, Komeda K, Ide T, Kubota N, Terauchi Y, Tobe K, Miki H, Tsuchida A, Akanuma Y, Nagai R, Kimura S, and Kadowaki T, J. Biol. Chem., 276, 41245-41254 (2001).

3) Forman BM, Ruan B, Chen J, Schroepfer GJ, and Evans RM, Proc. Natl. Acad. Sci. USA, 94, 10588-10593 (2001).

4) Martin G, Duez H, Blanquart C, Berezowski V, Poulain P, Fruchart JC, Najib-Fruchart J, Glineur C, and Staels B, J. Clin. Invest., 107, 1423-1432 (2001).

5) Argmann CA, Edwards JY, Sawyez CG, O'Neil CH, Hegele RA, Pickering JG, and Huff MW, J. Biol. Chem., 280, 2221222221 (2005)

6) Yano M, Matsumura T, Senokuchi T, Ishii N, Murata Y, Taketa K, Motoshima H, Taguchi T, Sonoda K, Kukidome D, Takuwa Y, Kawada T, Brownlee M, Nishikawa T, and Araki E, Circ. Res., 100, 1442-1451 (2007).

7) Takahashi N, Kawada T, Goto T, Yamamoto T, Taimatsu A, Matsui N, Kimura K, Saito M, Hosokawa M, Miyashita K, and Fushiki T, FEBS Lett., 514, 315-322 (2002).

8) Hohsaka T, Muranaka N, Komiyama C, Matsui K, Takaura S, Abe R, Murakami H, and Sisido M, FEBS Lett., 560, 173-177 (2004).

9) Taki M, Hohsaka T, Murakami H, Taira K, and Sisido M, J. Am. Chem. Soc., 124, 14586-14590 (2002).

10) Hohsaka T, Ashizuka Y, Taira H, Murakami H, and Sisido M, Biochemistry, 40, 11060-11064 (2001).

11) Abe R, Shiraga K, Ebisu S, Takagi H, and Hohsaka T, J. Biosci. Bioeng., 110, 32-38 (2010).

12) Taira H, Matsushita Y, Kojima K, Shiraga K, and Hohsaka T, Biochem. Biophys. Res. Commun., 374, 304-308 (2008).

13) Shimomura O, Johnson FH, and Saiga Y, J. Cell. Comp. Physiol., 59 223-239 (1962). 
14) Chalfie M, Tu Y, Euskirchen G, Ward WW, and Prasher DC, Science, 263, 802-805 (1994).

15) Souchier C, Ffrench M, Benchaib M, Catallo R, and Bryon PA, Cytometry, 20, 203-211 (1995).

16) Hayashida $\mathrm{O}$ and Uchiyama M, Tetrahedron Lett., 47, 40914094 (2006).

17) Takahashi N, Kawada T, Yamamoto $T$, Goto $T$, Taimatsu A, Aoki N, Kawasaki H, Taira K, Yokoyama KK, Kamei Y, and Fushiki T, J. Biol. Chem., 277, 16906-16912 (2002).

18) Oberkofler H, Esterbauer H, Linnemayr V, Strosberg AD, Krempler F, and Patsch W, J. Biol. Chem., 277, 16750-16757 (2005).
19) Kamei $Y$, Ohizumi H, Fujitani $Y$, Nemoto $T$, Tanaka $T$, Takahashi N, Kawada T, Miyoshi M, Ezaki O, and Kakizuka A, Proc. Natl. Acad. Sci. USA, 100, 12378-12383 (2003).

20) Yu C, Chen L, Luo H, Chen J, Cheng F, Gui C, Zhang R, Shen J, Chen K, Jiang H, and Shen X, Eur. J. Biochem., 272, 386-397 (2004).

21) Takahashi N, Goto T, Taimatsu A, Egawa K, Katoh S, Kusudo K, Sakamoto T, Ohyane T, Lee JY, Kim Y, Uemura T, Hirai S, and Kawada T, Biochem. Biophys. Res. Commun., 390, 13721376 (2002).

22) Hotamisligil GS, Johnson RS, Distel RJ, Ellis R, Papaioannou VE, and Spiegelman BM, Science, 274, 1377-1379 (1996). 